W.D. Oswald

Der 5. Kongress der Deutschen Gesellschaft für Gerontologie und Geriatrie vom 18.-20. Dezember 2000 in Nürnberg stand unter dem Motto „Altern im 21. Jahrhundert - Fakten und Visionen“. Um diesem Anspruch gerecht $\mathrm{zu}$ werden, wurden diesmal statt einer Plenarveranstaltung sechs durchgeführt, die sich interdisziplinär den derzeit wichtigsten Problemen der Gerontologie widmeten:

Prof. Dr. W.D. Oswald ( Institut für Psychogerontologie

Universität Erlangen-Nürnberg

Nägelsbachstr. 25

91052 Erlangen

\title{
5. Kongress der Deutschen Gesellschaft für Gerontologie und Geriatrie
}

1. K. Beyreuther und R. Hirsch „Alzheimer-Schicksal oder Herausforderung?"

2. G. Igl und G.M. Backes „Reiche Alte - arme Junge? Perspektiven zur Rentenfinanzierung"

3. I. Füsgen und T. Klie „Lebensverlängerung um jeden Preis"

4. C. Tesch-Römer „Ageing in (Central) Europe - European Ageing: Health and Care Systems and Intergenerational Solidarity in an European Perspective“

5. R. Schmidt, D. Schaeffer „Und wenn man pflegebedürftig wird, was dann?"

6. A. Niederfranke, Th. Nikolaus „Aktiv altern: eine Garantie für Gesundheit und Zufriedenheit?"
Leider ist es kurzfristig nicht gelungen, alle Beiträge für Heft 1/01 und Heft 2/02 zu gewinnen. Um die Aktualität $\mathrm{zu}$ erhalten, erscheint es jedoch durchaus als richtig, auf Vollständigkeit zu verzichten. In Heft 01 erschienen deshalb schwerpunktmäßig die Beiträge zu 4 und 6, in Heft 02 nun schwerpunktmäßig zu 1, 3 und 5. Den Perspektiven der Rentenfinanzierung wird ein eigenes Heft gewidmet werden.

Ich freue mich, dass damit ein repräsentativer Querschnitt der Diskussionen auf dem Nürnberger Kongress einer breiteren Öffentlichkeit zugänglich gemacht werden kann und danke allen beteiligten Moderatoren und Autoren für die rasche Erstellung ihrer Manuskripte. 